\title{
Estudio de las propiedades psicométricas de la Escala Barratt de Impulsividad (BIS-11) en niños y adolescentes
}

\section{Study of the psychometric properties of the Barratt Impulsiveness Scale (BIS-11) in children and adolescents}

\author{
Nicolás Chahín Pinzón \\ Facultad de Psicología. Universidad Manuela Beltrán. Bucaramanga, Colombia \\ Clara Eugenia Moncada Duarte \\ Facultad de Psicología. Universidad Manuela Beltrán. Bucaramanga, Colombia \\ Hadder Uriel Acosta Salazar \\ Corporación Universitaria Minuto de Dios. Bucaramanga, Colombia
}

Rec (12 de junio de 2018) Acept (17 de abril de 2019)

\begin{abstract}
Resumen
El objetivo de este estudio fue identificar y confirmar la estructura trifactorial de la Escala Barratt de Impulsividad (BIS-11) propuesta para adultos (Patton, Stanford y Barratt, 1995) en una muestra de 600 niños y adolescentes entre 9 y 17 años de edad $(M=12.9$, DT $=2.3)$. Para el análisis factorial los datos fueron divididos aleatoriamente en dos. Con la primera submuestra se realizó el Análisis Factorial Exploratorio, en el que se identificaron tres dimensiones. Posteriormente, con la segunda submuestra se llevó a cabo el Análisis Factorial Confirmatorio. La solución factorial resultante de 19 ítems arroja adecuados indicadores de ajuste $\left(\mathrm{CFI}=.909\right.$, IFI $=.912$, RMSEA $\left.=.043 \mathrm{CI}[.032-.054], \chi^{2} / g l=1.561\right)$. El alfa Cronbach total ha sido $\alpha=$.795. Las escalas obtienen coeficientes aceptables para estas edades, Impulsividad Atencional con $\alpha=.641$, Impulsividad Motora $\alpha=.659$ e Impulsividad no Planificadora $\alpha=.727$.

Palabras clave: Escala Barratt de Impulsividad Versión 11, impulsividad, análisis factorial, niños, adolescentes.
\end{abstract}

\begin{abstract}
The aim of this study was to identify and confirm the trifactorial structure of the Barratt Impulsiveness Scale (BIS-11) proposed for adults by Patton, Stanford and Barratt (1995) in a sample of children and adolescents. The sample consisted of 600 children between of 9 and 17 years old $(M=12.9, S D=2.3)$. For the factorial analysis the data were divided into two random samples. In the first subsample the Exploratory Factorial Analysis was performed, and three dimensions were identified. Subsequently, in the second subsample, Confirmatory Factor Analysis was performed. The factorial solution of 19 items showed adequate fit $(\mathrm{CFI}=.909, \mathrm{IFI}=.912, \mathrm{RMSEA}=.043 \mathrm{CI}[.032-.054], \chi 2 / \mathrm{gl}=1.561)$. The total Cronbach alpha was $\alpha=.795$. Each scale has acceptable coefficients: Attentional Impulsiveness $\alpha=.641$, Motor Impulsiveness $\alpha=.659$ and Non-planning Impulsiveness $\alpha=.727$.

Keywords: Barratt Impulsiveness Scale Version 11, impulsivity, factorial analysis, children, adolescents.
\end{abstract}




\section{Introducción}

En la actualidad existe un marcado interés por el estudio de la impulsividad, aunque no se ha llegado a un consenso general sobre su definición ni de los sustratos que la componen (Arce \& Santisteban, 2006; Riaño-Hernández, Guillen \& Buela-Casal, 2015; Stahl et al., 2013). No obstante, un creciente número de investigadores y clínicos concuerdan en incluir en su conceptualización dimensiones relacionadas con la falta de planificación, dificultades en mantener el foco de atención y la propensión a actuar antes de pensar (Barratt, 1985, 1993; Barratt \& Patton, 1983; Buss \& Plomin, 1975; Dickman, 1990; Moeller, Barratt, Dougherty, Schmitz \& Swann, 2001).

En niños y adolescentes el estudio y evaluación de la impulsividad es un elemento clave para poder comprender los efectos que este rasgo de personalidad tiene sobre la conducta, la regulación emocional y la adecuada adaptación al medio familiar, escolar y social. En tal sentido, la falta de control sobre los impulsos se encuentra asociada a gran cantidad de trastornos mentales en estas edades (American Psychiatric Association, 2013; Bridge et al., 2015; Chahín-Pinzón \& Briñez, 2011, 2015, 2018; Chahín-Pinzón, Lorenzo-Seva \& Vigil-Colet, 2012; Chahín-Pinzón, Reyes \& Vargas, 2017; Dir, Coskunpinar \& Cyders, 2014; Gibbons et al., 2016; Leeman, Hoff, Krishnan-Sarin, Patock-Peckham \& Potenza, 2014; Moncada \& Chahín-Pinzón, 2019; Secades-Villa, Martinez-Loredo, Grande-Gosende \& Fernández-Hermida, 2016).

A pesar de lo anterior, existen hoy en día muy pocos instrumentos de autoreporte debidamente validados que miden impulsividad en estas edades. El primero, es el Inventario de Impulsividad de Dickman para niños (DII-c), que se intentó adaptar para niños, pero sus propiedades psicométricas fueron bastante pobres, por tanto su administración no es recomendable para esta población (Brunas-Wagstaff, Tilley, Verity, Ford \& Thompson, 1997; Cosi, Morales-Vives, Canals, Lorenzo-Seva \& Vigil-Colet, 2008). El segundo, es el Cuestionario de Impulsividad I6 Junior (I6), que ha tenido adecuadas características psicométricas en muestras pertenecientes a culturas y lenguajes diferentes (Eysenck \& Eysenck, 1980; Saklofske \& Eysenck, 1983; Silva, Martorell \& Clemente, 1987; Stadler \& Janke, 2003).

El tercero, es la Escala Barratt de Impulsividad (BIS-11), con más de 55 años de historia y trasformaciones. Su versión actual para adultos posee tres factores: Impulsividad Atencional, Impulsividad Motora e Impulsividad no Planificadora (Patton, Stanford \& Barratt, 1995). A pesar de lo anterior, la identificación de esta solución factorial en muchos casos ha sido problemática. En este sentido, la escala que más fácilmente ha sido identificada es Impulsividad no Planificadora, y la más inestable es Impulsividad Atencional, pues sus ítems tienden a tener cargas factoriales repartidas en las otras dos escalas. Esta situación se hace mucho más evidente cuando se trabaja con muestras de niños y adolescentes, en donde dicha estructura ha sido más difícil de hallar, pues al parecer los componentes motores, cognitivos y atencionales del rasgo no se encuentran bien diferenciados en estas edades (Chahín-Pinzón, 2015a; Reise, Moore, Sabb, Brown \& London, 2013; Stanford, et al., 2009; Vasconcelos, Malloy-Diniz \& Correa, 2012; Pechorro, Andershed, Ray, Maroco \& Gonçalves, 2015).

Ante esta dificultad, la presente investigación intenta identificar la misma estructura trifactorial del BIS-11A original en la muestra de niños y adolescentes seleccionada para el presente estudio. Para el logro de este objetivo se partió de los ítems de la adaptación colombiana de Chahín, Cosi, Lorenzo-Seva y Vigil-Colet (2010), basada en la versión española para niños que Cosi, Vigil-Colet y Canals (2008) elaboraron sobre el instrumento que Fossati, Barratt, Acquarini \& Di Ceglie (2002) desarrollaron para adolescentes italianos, teniendo como referente la versión para adultos de Patton et al (1995).

Si esta estructura de Patton et al. (1995) es encontrada a través del análisis factorial de los ítems, se procederá a determinar la consistencia interna de las escalas, y se comprobará si existen diferencias entre los puntajes de las medias en los grupos de sexo y edad. Finalmente, se verificará si existe validez convergente y/o divergente, al correlacionar sus escalas con otros dos instrumentos que evalúan impulsividad: el Cuestionario de Impulsividad I6 Junior y el BIS 11-c. 


\section{Método}

\section{Participantes}

Muestra conformada por 600 escolares en edades comprendidas entre los 9 y 17 años $(M=12.9$, DT $=2.3)$, pertenecientes a la zona metropolitana de Bucaramanga (Colombia-Sur América), de los cuales 298 son mujeres y 302 varones.

\section{Instrumentos}

Escala Barratt de impulsividad para niños (BIS 11-c) Elaborada por Chahín et al. (2010) para niños y adolescentes colombianos. Se encuentra compuesto por 26 ítems y tres factores. El primero, Impulsividad Cognitiva con 5 ítems $(\alpha=.59)$, asociado a pensar y tomar decisiones de forma rápida. En este instrumento el factor es considerado como impulsividad funcional (v. gr. "Pienso con rapidez"). El segundo, Impulsividad Motora con 13 ítems $(\alpha=.74)$ referido a la tendencia actuar sin pensar ("Actúo sin pensar"). Y el tercero, Impulsividad no Planificadora con 8 ítems $(\alpha=.72)$, relacionado con la falta de planificación (v. gr. "Organizo mis actividades"). Las preguntas poseen cuatro alternativas de respuesta $(0=$ Nunca/Casi nunca; $1=$ Algunas veces; $2=$ Frecuentemente, $3=$ Siempre $/$ Casi siempre). El rango de la puntuación total se encuentra entre 0 y 78 .

Cuestionario I6 Junior de Impulsividad (I6) Esta es la adaptación que Chahín-Pinzón (en revisión) desarrolló para niños y adolescentes colombianos, a partir de la versión que Silva, et al. (1987) realizaron del I5 Junior de Eysenck, Pearson, Easting \& Allsopp (1985). El cuestionario posee 17 ítems y mide dos dimensiones. La primera, Impulsividad con 9 ítems $(\alpha=.67)$ relacionada con el sustrato de impulsividad general (v. gr. "¿Con frecuencia dices o haces cosas sin haberlo pensado antes?"). La segunda, Búsqueda de Aventura con 8 ítems $(\alpha=.74)$ asociada con la búsqueda de nuevas sensaciones y toma de riesgos premeditados (v. gr. "¿Te gusta mucho arriesgarte?"). El tipo de respuesta es dicotómico (Si-No). El rango de puntuación total se encuentra entre 0 y 17.

\section{Procedimiento}

Por ser una muestra de niños y adolescentes, inicialmente se contactó con las directivas de los centros educativos para obtener todas las autorizaciones necesarias. Los participantes respondieron los cuestionarios en las aulas habituales donde reciben sus clases, bajo la supervisión del investigador y el profesor de turno. El número promedio de estudiantes en cada salón de clase fue 30. El tiempo asignado para responder los tres instrumentos fue 25 minutos. Los procedimientos utilizados garantizaron el total anonimato y confidencialidad de los datos obtenidos.

\section{Análisis de datos}

La muestra fue dividida aleatoriamente en dos partes, para analizar la estructura factorial. Con la primera submuestra se efectuó el Análisis Factorial Exploratorio (AFE), en donde se utilizó el método de extracción de componentes principales con rotación Promax. La adecuación de la muestra fue determinada mediante el test de Kaiser-Meyer-Olkin y el test de esfericidad de Barlett.

Con la segunda submuestra se realizó el Análisis Factorial Confirmatorio (AFC) de la solución factorial hallada en la primera submuestra. Para la estimación de los parámetros se utilizó el procedimiento de estimación de máxima verosimilitud. Los referentes para evaluar el ajuste al modelo fueron los criterios propuestos por $\mathrm{Hu}$ \& Bentler (1999). En este sentido, como índices de ajuste relativo se utilizaron el IFI (Incremental Fit Index) y el CFI (Comparative Fit Index). Y como índices de ajuste absoluto el RMSEA (Root Mean Square Error of Approximation) y la razón $\chi^{2} / g l$. Si bien no hay consenso sobre estos índices, se consideran aceptables valores iguales o superiores a .9 para el CFI y el IFI. Y con respecto al RMSEA, son aceptables los inferiores a .08 y excelentes los que poseen valores inferiores a .04. Y para la razón $\chi^{2} / g l$ son indicadores de buen ajuste los inferiores a 2.0 (Bentler, 1990; Fan \& Sivo, 2007; Hu \& Bentler, 1995). 
Para determinar la correlación entre los factores y la validez convergente y/o divergente se calculó el coeficiente $r$ de Pearson. La fiabilidad fue estimada con el alfa de Cronbach. La prueba $t$ de Student y el tamaño del efecto de Cohen fueron utilizadas para analizar las diferencias entre los respectivos grupos de sexo y edad. El análisis de los datos fue realizado con los programas SPSS 18.0 y AMOS 18.0.

\section{Resultados}

\section{Análisis Factorial Exploratorio}

Previo al inicio del procedimiento del análisis factorial, se excluyeron tres de los cinco ítems correspondientes a la escala de Impulsividad Cognitiva. El primero de ellos, que fue redactado y agregado exclusivamente para construir esta escala ("Cuando mis amigos me preguntan algo, puedo responder rápidamente") (Cosi, Vigil-Colet \& Canals, 2008). Y los otros dos, por ser muy similares en su redacción a los ítems propuestos por Luengo, Carrillo-de-la-Peña \& Otero (1991) para Impulsividad Cognitiva ("Decido rápidamente”. "Pienso con rapidez").

Con los 23 ítems restantes se procedió a realizar el AFE con la primera submuestra $(\mathrm{N}=300)$. En esta solución factorial la totalidad de los ítems obtuvieron cargas factoriales superiores a .35 en su respectivo factor y saturaciones inferiores a . 3 en los demás . Los índices de adecuación muestral estuvieron dentro de los parámetros, con $\mathrm{KMO}=.757$ y una prueba esfericidad de Bartlett significativa $\left(\chi^{2}(253)=1057.394, p<.0001\right)$. La solución resultante puede verse en la Tabla 1.

Tabla 1. Ítems y solución resultante del análisis factorial exploratorio

\begin{tabular}{|c|c|c|c|c|}
\hline & \multirow[b]{2}{*}{ Ítems } & \multicolumn{3}{|l|}{ Escala } \\
\hline & & Atencional & Motora & No Planificadora \\
\hline 14 & $\begin{array}{l}\text { En el colegio, soy de los primeros en levantar la mano cuando el } \\
\text { profesor hace una pregunta. * }\end{array}$ & .648 & & \\
\hline 24 & Cuando estoy pensando en algo me distraigo con facilidad. ** & .615 & & \\
\hline 17 & $\begin{array}{l}\text { Cuando estoy haciendo algo que requiere concentración, me } \\
\text { distraigo con facilidad. }\end{array}$ & .579 & & \\
\hline 9 & Me puedo concentrar rápidamente. * & .555 & & \\
\hline 5 & Me cuesta trabajo estar atento. & .448 & & \\
\hline 25 & Me cuesta trabajo quedarme quieto en clase. & .395 & & \\
\hline 16 & Actúo sin pensar. & & .630 & \\
\hline 15 & Cambio con facilidad mi manera de pensar. & & .520 & \\
\hline 13 & Digo cosas sin pensar. & & .512 & \\
\hline 18 & Me dejo llevar por mis impulsos. & & .495 & \\
\hline 8 & Me desespero con facilidad. & & .446 & \\
\hline 2 & Hago las cosas sin pensarlas. & & .443 & \\
\hline 21 & Compro cosas sin pensar. & & .409 & \\
\hline 20 & Cambio con frecuencia de amigos. & & .397 & \\
\hline 23 & Gasto más de lo que tengo. & & .379 & \\
\hline 11 & Me gusta pensar bien las cosas.* & & & .701 \\
\hline 26 & Organizo mis actividades. * & & & .699 \\
\hline 19 & Me gusta pensar las cosas. * & & & .680 \\
\hline 7 & Organizo mi tiempo libre.* & & & .609 \\
\hline 12 & Hago planes para el futuro. & & & .562 \\
\hline 1 & Planeo las cosas que hago.* & & & .544 \\
\hline 22 & Soluciono los problemas uno por uno. * & & & .517 \\
\hline 10 & Ahorro lo que más puedo. * & & & .367 \\
\hline
\end{tabular}




\section{Análisis factorial confirmatorio}

Con la segunda submuestra $(\mathrm{N}=300)$ se efectuó el AFC de la solución factorial resultante del AFE. En un primer análisis la estructura factorial para los 23 ítems arrojó unos pobres índices de ajuste $(\mathrm{CFI}=.825, \mathrm{IFI}=.833$, $\left.\mathrm{RMSEA}=.052 \mathrm{CI}[.044, .060], \chi^{2} / g l=1.807\right)$. Para mejorar el modelo y obtener indicadores aceptables, se eliminaron los ítems que poseían cargas de regresión estandarizadas inferiores a .3. Como resultado de este proceso se descartaron cuatro ítems. Uno de la escala Impulsividad Atencional ("En el colegio, soy de los primeros en levantar la mano cuando el profesor hace una pregunta"), dos de Impulsividad Motora ("Cambio con facilidad mi manera de pensar". "Cambio con frecuencia de amigos") y uno de Impulsividad No Planificadora ("Hago planes para el futuro").

Como se exhibe en la Tabla 2, la solución resultante de 19 ítems $(\mathrm{CFI}=.909, \mathrm{IFI}=.912$, RMSEA $=.043 \mathrm{CI}$ $\left.[.032-.054], \chi^{2} / g l=1.561\right)$ obtuvo unos mejores indicadores de ajuste, en comparación con la de 23 ítems. Además, sus cargas de regresión estandarizadas presentan para todos los ítems valores superiores a .3 (Figura 1).

Tabla 2. Indicadores de ajuste para los dos modelos analizados

\begin{tabular}{lllllllll}
\hline \multirow{2}{*}{ Modelo } & \multicolumn{2}{l}{ Índices absolutos } & \multicolumn{2}{l}{$\chi^{2} / g l$} & RMSEA & IC $^{*}$ & CFI & IFI \\
\cline { 2 - 8 } & $\chi^{2}$ & $g l$ & $p$ & 1.807 & .052 & .044 & .825 & .833 \\
\hline 23 ítems & 410.132 & 227 & .000 & & & -.060 & & \\
\hline 19 ítems & 232.569 & 149 & .000 & 1.561 & .043 & .032 & .909 & .912 \\
\hline
\end{tabular}

*Intervalo de confianza al 95\%

Figura 1. Modelo resultante del análisis factorial confirmatorio con 19 items

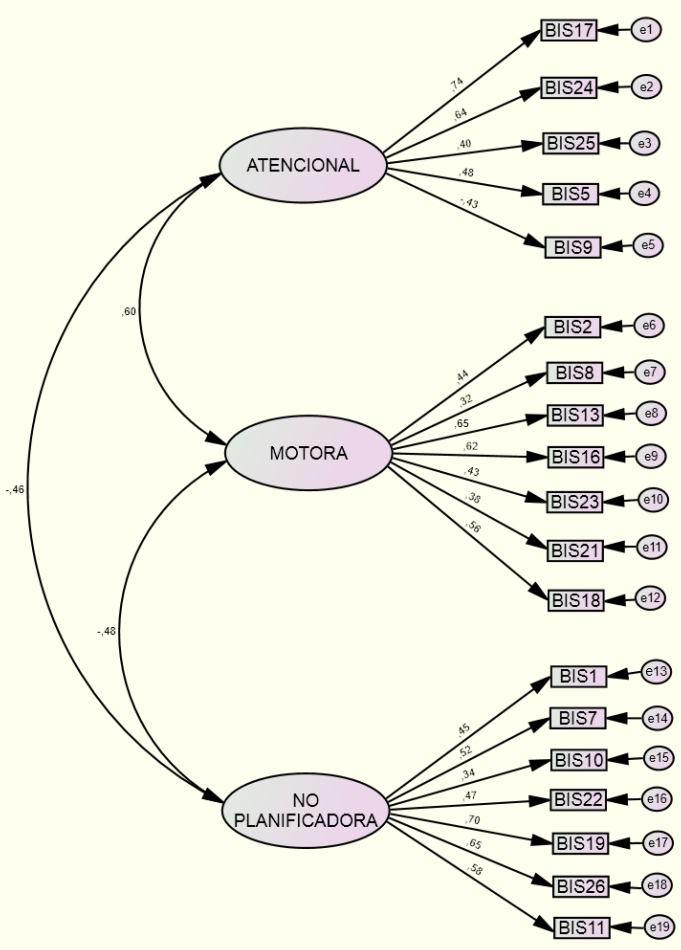




\section{Estadísticos descriptivos}

Los valores de la media para los grupos de sexo y edad para cada una de las tres escalas: Impulsividad Atencional, Impulsividad Motora e Impulsividad no Planificadora, se pueden observar en la Tabla 3.

Tabla 3. Descriptivos, $t$ de Student y tamaño del efecto por sexo y edad

\begin{tabular}{|c|c|c|c|c|c|c|c|c|}
\hline \multirow{3}{*}{ Escalas } & \multicolumn{4}{|c|}{ Grupos de sexo } & \multirow[b]{3}{*}{$T$} & \multirow[b]{3}{*}{$\mathrm{gl}$} & \multirow[b]{3}{*}{$\mathrm{p}$} & \multirow[b]{3}{*}{$d$} \\
\hline & \multicolumn{2}{|c|}{ Mujeres } & \multicolumn{2}{|c|}{ Varones } & & & & \\
\hline & Media & $\mathrm{DE}$ & Media & $\mathrm{DE}$ & & & & \\
\hline Atencional & 5.01 & 2.95 & 4.79 & 2.82 & .915 & 569 & .361 & - \\
\hline Motora & 6.02 & 3.40 & 6.00 & 3.58 & .037 & 549 & .971 & - \\
\hline \multirow[t]{2}{*}{ No planificadora } & 7.87 & 4.29 & 8.03 & 4.21 & .453 & 552 & .650 & - \\
\hline & \multicolumn{4}{|c|}{ Grupos de edad } & & & & \\
\hline \multirow[t]{2}{*}{ Escalas } & \multicolumn{2}{|c|}{ 9-13 años } & \multicolumn{2}{|c|}{ 14-17 años } & & & & \\
\hline & Media & DE & Media & $\mathrm{DE}$ & $T$ & $\mathrm{gl}$ & $\mathrm{p}$ & $d$ \\
\hline Atencional & 4.67 & 2.85 & 5.23 & 2.90 & 2.263 & 568 & .024 & .05 \\
\hline Motora & 5.80 & 3.59 & 6.30 & 3.34 & 1.684 & 548 & .093 & - \\
\hline No planificadora & 7.32 & 4.32 & 8.77 & 4.01 & 4.043 & 551 & .000 & .25 \\
\hline
\end{tabular}

\section{Comparaciones entre grupos de sexo y edad}

$\mathrm{Al}$ realizar las comparaciones entre mujeres y varones no se hallaron diferencias significativas en las tres escalas. Sin embargo, como se aprecia en la Tabla 3, en los grupos de edad, la media de Impulsividad Atencional fue mayor en el grupo de 14-17 años $(t=2.263, p<.05 ; d=.05)$, lo mismo ocurrió con Impulsividad no Planificadora, pero con un tamaño del efecto mayor $(t=4.043, p<.001 ; d=.25)$.

\section{Fiabilidad}

Como se exhibe en la Tabla 4, la consistencia interna para el total del instrumento sido de $\alpha=.795$. Por su parte, las tres escalas han arrojado valores superiores a .6. En este sentido Impulsividad Atencional ha presentado el menor valor $(\alpha=.641)$, mientras que Impulsividad Motora e Impulsividad no Planificadora han tenido coeficientes superiores $(\alpha=.659 ; \alpha=.727)$.

Con referencia a la fiabilidad obtenida en mujeres y varones, no se encontraron diferencias significativas con el estadístico de contraste $w$ de Feldt (1969), aunque los valores son ligeramente superiores en los hombres. $\mathrm{Y}$ en los dos grupos de edad, tampoco hubo diferencias, no obstante la consistencia es mayor en el grupo de $14-17$ años. 
Tabla 4. Consistencia interna e intervalos de confianza al 95\% por sexo y edad

\begin{tabular}{llllll}
\hline Escala & Mujeres & Varones & $9-13$ años & $14-17$ años & Total \\
\hline Atencional & $.659(.593-.718)$ & $.623(.549-.688)$ & $.635(.538-.716)$ & $.681(.613-.740)$ & $.641(.592-.686)$ \\
Motora & $.660(.594-.718)$ & $.661(.596-.719)$ & $.657(.596-.712)$ & $.661(.591-.723)$ & $.659(.613-.701)$ \\
No & $.739(.689-.784)$ & $.716(.662-.764)$ & $.715(.664-.761)$ & $.730(.675-.779)$ & $.727(.691-.760)$ \\
$\begin{array}{l}\text { Planificadora } \\
\text { Total }\end{array}$ & $.800(.762-.834)$ & $.792(.753-, 828)$ & $.783(.744-.818)$ & $.804(.764-.840)$ & $.795(.768-.820)$ \\
\hline
\end{tabular}

\section{Correlación entre escalas y validez convergente}

En la Tabla 5 se puede ver que el coeficiente de Pearson es positivo entre las tres escalas de esta adaptación del BIS-11, encontrándose el mayor valor entre Impulsividad Atencional e Impulsividad Motora $(r=.46)$.

Tabla 5. Coeficientes de correlación de Pearson entre escalas

\begin{tabular}{llll}
\hline Escala & Atencional & Motora & No Planificadora \\
\hline Atencional & - & $.46^{* *}$ & $.30^{* *}$ \\
Motora & $.46^{* *}$ & - & $.31^{* *}$ \\
No Planificadora & $.30^{* *}$ & $.31^{* *}$ & - \\
\hline${ }^{* *} p<.01$. & & \\
\hline
\end{tabular}

Como se aprecia en la Tabla 6, las correlaciones de la actual versión del BIS-11 con las dimensiones del Cuestionario I6 Junior muestran que la escala Búsqueda de Aventura ha arrojado coeficientes relativamente bajos tanto para Impulsividad Motora $(r=.11)$ como para Impulsividad no Planificadora $(r=.18)$, además no hay una correlación significativa con Impulsividad Atencional. Por otra parte, la escala Impulsividad del Cuestionario I6 Junior, ha tenido correlaciones superiores con Impulsividad Atencional $(r=.40)$, Impulsividad Motora $(r=.55)$ e Impulsividad no Planificadora $(r=.42)$.

Por su parte, la escala Impulsividad Cognitiva del BIS 11-c de Chahín et al. (2010) ha arrojado todos los coeficiente de correlación negativos: Impulsividad Atencional $(r=-.37)$, Impulsividad Motora $(r=-.17) \mathrm{e}$ Impulsividad no Planificadora $(r=-.17)$. La escala Impulsividad Motora del BIS 11-c ha tenido correlaciones positivas bastante altas con Impulsividad Atencional $(r=.73)$ e Impulsividad Motora $(r=.88)$, mientras que con Impulsividad no Planificadora el valor ha sido menor $(r=.29)$. Finalmente, la escala Impulsividad no Planificadora del BIS 11-c ha correlacionado positivamente con Impulsividad Atencional $(r=.28)$ e Impulsividad motora $(r=.30)$, presentando el mayor coeficiente con Impulsividad no Planificadora $(r=.97)$ (Tabla 6).

Con respeto a la edad, se ha encontrado una baja correlación con Impulsividad Atencional $(r=.09) \mathrm{e}$ Impulsividad Motora $(r=.10)$, pero con Impulsividad no Planificadora el valor ha sido mayor $(r=.21)$. Un comportamiento muy similar se observa en las correlaciones con el grado de estudios (Tabla 6). 
Tabla 6. Correlación del instrumento con otras dimensiones de impulsividad

\begin{tabular}{lllll}
\hline & \multicolumn{5}{l}{ Actual Versión } \\
\hline Instrumento & Escala & Atencional & Motora & No Planificadora \\
\hline \multirow{2}{*}{ I6 Junior Eysenck } & Aventura & .050 & $.11^{*}$ & $.18^{* *}$ \\
& Impulsividad & $.40^{* *}$ & $.55^{* *}$ & $.42^{* *}$ \\
\hline \multirow{2}{*}{ Versión BIS11-c } & Cognitiva & $-.37^{* *}$ & $-.17^{* *}$ & $-.17^{* *}$ \\
Chahín et al. $(2010)$ & Motora & $.73^{* *}$ & $.88^{* *}$ & $.29^{* *}$ \\
& No planificadora & $.28^{* *}$ & $.30^{* *}$ & $.97^{* *}$ \\
\hline & Edad & $.09^{*}$ & $.10^{*}$ & $.21^{* *}$ \\
& Grado & .05 & .06 & $.21^{* *}$ \\
\hline${ }^{*} p<.05 ; * * p<.01$. & & & & \\
\hline
\end{tabular}

\section{Discusión}

Aunque la mayoría de estudios previos con niños y adolescentes informan que la estructura del BIS-11 identificada para adultos por Patton et al.(1995) no siempre es encontrada en el análisis factorial (Chahín-Pinzón, 2015b; Cosi, Vigil-Colet, Canals \& Lorenzo-Seva, 2008; Hartmann, Rief \& Hilbert, 2011; Fossati et al., 2002; Martínez-Loredo, Fernández-Hermida, Fernández-Artamendi, Carballo \& García-Rodríguez, 2015; Salvo \& Castro, 2013; Von Diemen, Szobot, Kessler y Pechansky, 2007), los resultados de esta adaptación colombiana indican que esta misma estructura de Patton et al. (1995) con sus tres dimensiones, Impulsividad Atencional, Impulsividad Motora e Impulsividad no Planificadora, sí se ha podido identificar y confirmar para una solución factorial con 19 ítems $\left(\mathrm{CFI}=.909, \mathrm{IFI}=.912, \mathrm{RMSEA}=.043 \mathrm{CI}[.032-.054], \chi^{2} / g l=1.561\right)$. Correspondiendo la totalidad del contenido de cada uno de los ítems a la conceptualización teórica tridimensional del constructo impulsividad propuesto por estos autores.

Es conveniente tener en cuenta que en la actualidad existen sólo dos adaptaciones de que han informado de adecuados indicadores de ajuste al modelo trifactorial en este rango de edades. La primera, fue la realizada por Recio (2012) con un instrumento de 27 ítems, con una muestra de niños españoles con edades similares a las de este estudio (9 - 16 años), partió de la versión realizada por Oquendo et al. (2001). Y el segunda, es una versión china de 30 ítems para adolescentes, pero la media de la edad de su muestra fue superior a 17 años (Wan, Zhang, Jin \& Cheng, 2016).

La consistencia interna para el total de los ítems de esta versión ha sido buena en comparación con lo que se reporta para estas edades $(\alpha=.795)$, mucho más si se tiene en cuenta que la presente versión posee un 33\% menos de ítems que la original. Al comparar este coeficiente con otras versiones para niños y adolescentes, independientemente de la estructura factorial reportada, ha sido bastante similar al de otras adaptaciones (Fossati et al., 2002; Hartmann et al., 2011; Recio, 2012; Von Diemen et al., 2007; Yao et al., 2007). En lo que concierne a la fiabilidad de las escalas, el alfa Cronbach para cada una de ellas ha estado por encima del punto de corte: Impulsividad Atencional ( $\alpha=.641)$, Impulsividad Motora $(\alpha=.659)$, e Impulsividad no Planificadora $(\alpha=.727)$, por tanto han arrojado coeficientes bastante aceptables para estas edades, a pesar de que todas las escalas poseen comparativamente menos ítems que las otras versiones. Estos valores son bastante similares e incluso superiores a los informados por la versión española de Recio (2012) en sus tres escalas $(\alpha=.65, \alpha=.67, \alpha=.63)$. Pero han sido inferiores a los reportados para la adaptación china de Wan et al. (2016) $(\alpha=.67, \alpha=.80, \alpha=.85)$, más en este caso hay que recordar que la media de la edad en esta muestra es mucho mayor y no incluye niños. Además, de acuerdo al estadístico de contraste $w$ de Feldt, no se hallaron diferencias significativas en la fiabilidad entre mujeres y varones en las tres dimensiones, resultado que refleja la estabilidad de esta medida entre sexos. Y por otra parte, es importante remarcar un aspecto poco frecuente, referido a la edad en este tipo de estudios con niños y adolescentes. Es que la fiabilidad en los grupos de edad, además de exhibir coeficientes aceptables, de acuerdo al estadístico $w$ de Feldt no ha informado de diferencias significativas entre los dos grupos estudiados 
(9-13 años, 14-17 años). Lo anterior es un buen indicador de la estabilidad que posee esta adaptación.

Es pertinente mencionar en este punto, que la consistencia interna de las escalas obtenidas este estudio podría mejorar sensiblemente, si en investigaciones futuras se retomaran los ítems que fueron eliminados por Cosi, Vigil-Colet, Canals \& Lorenzo-Seva (2008) de la adaptación italiana para adolescentes que realizó Fossati et al., (2002). Y si adicionalmente se realizara una nueva adaptación de los ítems para niños y adolescentes a partir de la versión original de Patton et al. (1995). Teniendo esta vez mucho más presente los aspectos relacionados con el desarrollo evolutivo y las conductas en que se manifiesta el rasgo impulsivo en estas edades en la actualidad (Chahín-Pinzón, 2014; Hambleton, 2005)

Al comparar las medias de los grupos de sexo, se pudo apreciar que no hay diferencias significativas, lo cual se encuentra en línea con lo reportado en estudios previos, relacionados con la invariancia de este rasgo entre mujeres y varones (Patton et al., 1995; Recio, 2012; Stanford, et al., 2009). No obstante, en los grupos de edad, llama la atención que quienes tenían una mayor edad y se encontraban viviendo la etapa de la adolescencia propiamente dicha (14-17 años), exhibieron puntajes mayores en Impulsividad Atencional $(d=.05)$, pero principalmente en Impulsividad no Planificadora $(d=.25)$, lo anterior se confirma con los hallazgos reportados en estudios previos (Chahín, 2013; Recio, 2012; Stanford, et al., 2009). Esta diferencia a favor de los de mayor edad en estas dos dimensiones, asociadas con impulsividad disfuncional, es un factor de vulnerabilidad para la manifestación de conductas problemáticas en esta etapa de la vida (Chambers, Taylor \& Potenza, 2003; Martínez-Loredo et al., 2015).

Las correlaciones entre las tres escalas de esta adaptación han sido en todos los casos positivas. En especial, hay que tener en cuenta los coeficientes positivos resultantes entre Impulsividad Atencional con Impulsividad Motora $(r=.46)$ y con Impulsividad no Planificadora $(r=.30)$. Estos valores positivos se deben a que los ítems que conforman Impulsividad Atencional describen en su redacción aspectos referidos a la dificultad para lograr concentrarse o centrar la atención, que es uno de los sustratos de la impulsividad propuestos por Patton et al. (1995). Contrario a lo que ocurre con la escala Impulsividad Cognitiva del BIS 11-c de Chahín et al. (2010), cuyos ítems se encuentran asociados en su totalidad con impulsividad funcional. Y por tanto, las correlaciones resultantes de este instrumento son negativas con Impulsividad Motora e Impulsividad no Planificadora (Barratt, 1985; Chahín, 2013; Cosi, Vigil-Colet, Canals, 2008; Dickman, 1990).

En cuanto a la validez convergente y divergente, en primer término se ha visto que la escala Búsqueda de Aventura del Cuestionario I6 Junior ha arrojado correlaciones bastante bajas con Impulsividad Motora e Impulsividad no Planificadora $(r=.11, r=.18)$, pero no correlacionó con Impulsividad Atencional. Esto se encuentra en línea con el estudio de Barratt (1985), en donde señaló que los sustratos de la impulsividad del BIS no se encontraban relacionados con el atrevimiento. En segundo término, la escala Impulsividad del I6, sí ha arrojado mejores correlaciones con las tres dimensiones de esta versión, en Impulsividad Atencional $(r=.40)$, Impulsividad Motora $(r=.55)$ e Impulsividad no Planificadora $(r=.42)$, lo cual es un buen indicador de validez convergente.

Y con respecto a las correlaciones de la presente adaptación con las tres escalas del BIS 11-c de Chahín et al. (2010), se advierte en primera instancia que la escala Impulsividad Atencional ha correlacionado positivamente, tanto con Impulsividad Motora $(r=.73)$ como con Impulsividad no Planificadora $(r=.28)$, pero con Impulsividad Cognitiva el coeficiente ha sido negativo $(r=-.37)$. En segunda instancia, la Impulsividad Motora de este estudio ha tenido una alta correlación con la escala del mismo nombre del BIS 11-c ( $r=.88)$, y un menor valor con la Impulsividad no Planificadora $(r=.30)$. Más nuevamente se observa una correlación negativa con Impulsividad Cognitiva $(r=-.17)$. Y en tercera instancia, en la escala Impulsividad no Planificadora de la presente versión, las correlaciones han sido positivas tanto para Impulsividad Motora $(r=.29)$ como para Impulsividad no Planificadora $(r=.97)$, pero una vez más es negativa para Impulsividad Cognitiva $(r=-.17)$. Por lo visto, en las correlaciones de la presente adaptación del BIS-11con el BIS 11-c de Chahín et al. (2010), se aprecia que estos instrumentos poseen dos escalas que respectivamente miden prácticamente los mismos sustratos y poseen el mismo nombre, como lo son Impulsividad Motora e Impulsividad no Planificadora, lo que se ve reflejado en las altas correlaciones entre estas escalas $(r=.88, r=.97)$. No obstante, la diferencia fundamental de estos dos instrumentos se encuentra en la tercera dimensión, que para esta versión se refiere a aspectos disfuncionales de la impulsividad, como son la dificultad de focalizar la atención o de lograr concentrarse (Impulsividad Atencional). Y para el BIS 11-c con aspectos funcionales de la impulsividad, como son pensar o actuar rápidamente (Impulsividad Cognitiva). 
Es pertinente agregar, que al eliminar en el análisis factorial la escala Impulsividad Cognitiva y sustituirla por la escala Impulsividad Atencional, han mejorado sensiblemente las propiedades psicométricas del instrumento. Y esto se debe a que la escala Impulsividad Cognitiva históricamente ha sido muy problemática, tanto en su identificación como factor, como en su consistencia interna, obteniendo valores muy pobres en adultos $(\alpha=.27$ y $\alpha=.18)$ (Luengo, Carrillo de la Peña \& Otero, 1991). Y más concretamente, en niños y adolescentes, estos coeficientes han sido bajos, tanto en muestras colombianas como españolas $(\alpha=.59, \alpha=.60)$ (Chahín et al. 2010; Cosi, Vigil-Colet, Canals \& Lorenzo-Seva, 2008).

En conclusión, el Cuestionario BIS-11 para niños y adolescentes de este estudio posee adecuadas propiedades psicométricas en sus tres dimensiones. Además, tiene la ventaja de tener comparativamente un menor número de ítems, que permite que sea respondido muy rápidamente. Por tanto, es un instrumento válido, confiable y de muy fácil aplicación que puede ser utilizado para evaluar la impulsividad en el campo clínico, investigativo y educativo.

\section{Referencias}

American Psychiatric Association. (2013). Diagnostic and statistical manual of mental disorders (5th ed.). Washington, DC: Author. Arce, E., \& Santisteban, C. (2006). Impulsivity: A review. Psicothema, 18, 213-220.

Barratt, E. S. (1985). Impulsiveness subtraits: Arousal and information processing. En Spence, J. T., e Izard, C. E. (Eds.), Motivation, emotion and personality (pp.137-146). Amsterdam: Elsevier.

Barratt, E. S. (1993). Impulsivity: Integrating cognitive, behavioral, biological and environmental data. En McCowan, W., \& Shure, M. (Eds.), The impulsive client: Theory, research and treatment (pp. 39-56). Washington D.C.: American Psychological Association.

Barratt, E. S., \& Patton, J. H. (1983). Impulsivity: Cognitive, behavioral, and psychophysiological correlates. En Zuckerman M. (Ed.), Biological bases of sensation seeking, impulsivity and anxiety (pp. 77-121). Hillsdale, N. J.: Erlbaum.

Bentler, P. M. (1990). Comparative fit indexes in structural models. Psychological Bulletin, 107, 238-246. http://dx.doi.org/10.1037/00332909.107.2.238

Bridge, J. A., Reynolds, B., McBee-Strayer, S. M., Sheftall, A. H., Ackerman, J., Stevens, J., ... y Brent, D. A. (2015). Impulsive aggression, delay discounting, and adolescent suicide attempts: effects of current psychotropic medication use and family history of suicidal behavior. Journal of child and adolescent psychopharmacology, 25, 114-123. https://doi.org/10.1089/cap.2014.0042

Brunas-Wagstaff, J., Tilley, A., Verity, M., Ford, S., \& Thompson, D. (1997). Functional and dysfunctional impulsivity in children and their relationship to Eysenck's impulsiveness and venturesomeness dimensions. Personality and individual Differences, 22, $19-25$. https://doi.org/10.1016/S0191-8869(96)00173-0

Buss, A. H., \& Plomin, R. (1975). A temperament theory of personality development. New York: Wiley.

Chahín, N. (2013). Adaptación de dos instrumentos para niños colombianos: la escala Barratt de impulsividad (BIS-11C) y el cuestionario de agresividad de Buss y Perry (AQ). (Tesis doctoral) Universitat Rovira i Virgili. http://hdl.handle.net/10803/128182

Chahín, N., Cosi, S., Lorenzo-Seva, U., \& Vigil-Colet, A. (2010). Stability of the factorial structure of Barrat's Impulsivity Scales for children across cultures: a comparison between Spain and Colombia. Psicothema, 22, 983-989.

Chahín-Pinzón, N. (2014). Aspectos a tener en cuenta cuando se realiza una adaptación de test entre diferentes culturas. Psychologia: Avances de la Disciplina, 8, 107-110.

Chahín-Pinzón, N. (2015a). Revisión de las características psicométricas de la escala Barratt de impulsividad (BIS) a través de su historia: desde sus orígenes hasta la actualidad. Psicología desde el Caribe, 32, 315-326. http://dx.doi.org/10.14482/psdc.32.2.6110

Chahín-Pinzón, N. (2015b). Consideraciones y reflexiones acerca de la versión colombiana de la escala Barratt de impulsividad para niños (BIS-11c). Psicogente, 18, 396-405. http://doi.org/10.17081/psico.18.34.514

Chahín-Pinzón, N (en revisión). Propiedades psicométricas del Cuestionario de Impulsividad I6 Junior en niños y adolescentes. Terapia Psicológica.

Chahín-Pinzón, N., \& Briñez, B. (2011). Actividad física en adolescentes y su relación con agresividad, impulsividad, internet y videojuegos. Psychologia. Avances de la disciplina, 5, 1-16.

Chahín-Pinzón, N., \& Briñez, B. (2015). Propiedades psicométricas de la Escala de Ideología de Género en adolescentes colombianos. Universitas Psychologica, 14, 15-23. http://dx.doi.org/10.11144/Javeriana.upsy14-1.ppei

Chahín-Pinzón, N., \& Briñez, B. (2018). Propiedades psicométricas del Cuestionario de Adicción a Internet y Videojuegos para Adolescentes. Universitas Psychologica, 17. https://doi.org/10.11144/Javeriana.upsy17-4.ppca 
Chahín-Pinzón, N., Lorenzo-Seva, U., \& Vigil-Colet, A. (2012). Características psicométricas de la adaptación colombiana del Cuestionario de Agresividad de Buss y Perry en una muestra de preadolescentes y adolescentes de Bucaramanga. Universitas Psychologica, 11, 979-988.

Chahín-Pinzón, N., Reyes, J., \& Vargas, J. (2017). Aspectos psicológicos a tener en cuenta en la atención de víctimas de la trata de personas. Psychologia. Avances de la disciplina, 11, 121-129. http://dx.doi.org/10.21500/19002386.3107

Chambers, R. A., Taylor, J. R. \& Potenza, M. N. (2003). Developmental neurocircuitry of motivation in adolescence: A critical period of addiction vulnerability. The American Journal of Psychiatry, 160, 1041-1052. https://doi.org/10.1176/appi.ajp.160.6.1041

Cosi, S., Morales-Vives, F., Canals, J., Lorenzo-Seva, U., \& Vigil-Colet, A. (2008). Functional and dysfunctional impulsivity in childhood and adolescence. Psychological Reports, 103, 67-76. https://doi.org/10.2466/pr0.103.1.67-76

Cosi, S., Vigil-Colet, A., \& Canals, J. (2008). Desarrollo de la versión mejorada del BIS-11c: Relaciones con agresividad y rendimiento académico. Póster presentado en X Jornadas de la Sociedad Española para la Investigación de las Diferencias Individuales, Salamanca, España.

Cosi, S., Vigil-Colet, A., Canals, J., \& Lorenzo-Seva, U. (2008). Psychometric properties of the Spanish Adaptation of the Barratt Impulsiveness Scale- 11a for children. Psychological Reports, 103, 336-346.

Dickman, S. J. (1990). Functional and dysfunctional impulsivity, personality and cognitive correlates. Journal of Personality and Social Personality, 58, 95-102. http://dx.doi.org/10.1037/0022-3514.58.1.95

Dir, A. L., Coskunpinar, A., \& Cyders, M. A. (2014). A meta-analytic review of the relationship between adolescent risky sexual behavior and impulsivity across gender, age, and race. Clinical psychology review, 34, 551-562. https://doi.org/10.1016/j.cpr.2014.08.004

Eysenck, S. B. G, Pearson, P. R., Easting, G., \& Allsopp, J. F. (1985). Age norms for impulsiveness, venturesomeness and empathy in adults. Personality and individual differences, 6, 613-619. https://doi.org/10.1016/0191-8869(85)90011-X

Eysenck, S.B.G., \& Eysenck, H.J. (1980). Impulsiveness and venturesomeness in children. Personality and Individual Differences, 1 , 73-78. https://doi.org/10.1016/0191-8869(80)90006-9

Fan, X., \& Sivo, S. A. (2007). Sensitivity of fit indices to model misspecification and model types. Multivariate Behavioral Research, 42, 509-529. http://dx.doi.org/10.1080/00273170701382864

Feldt, L. S. (1969). A test of the hypothesis that Cronbach's alpha or Kuder-Richardson coefficient twenty is the same for two tests. Psychome trika, 34, 363-373. http://dx.doi.org/10.1007/BF02289364

Fossati, A., Barratt, E. S., Acquarini, E., \& Di Ceglie, A. (2002). Psychometric properties of and adolescent version of the Barratt Impulsiveness Scale-11 for a sample of Italian high school students. Perceptual and Motor Skills, 95, 621-635. http://dx.doi.org/10.2466/ PMS.95.5.621-635

Gibbons, F. X., Kingsbury, J.H., Wills, T. A., Finneran, S. D., Dal Cin, S., y Gerrard, M. (2016). Impulsivity moderates the effects of movie alcohol portrayals on adolescents' willingness to drink. Psychology of addictive behaviors, 30, 325-334. http://dx.doi. org/10.1037/adb0000178

Hambleton, R. K. (2005). Issues, designs and technical guidelines for adapting tests into multiple languages and cultures. En Hambleton, R. K.., Merenda, P. F., \& Spielberger, C. (Eds.), Adapting educational and psychological tests for crosscultural assessment (pp. 3-38). London: L.E.A

Hartmann, A. S., Rief, W., \& Hilbert, A. (2011). Psychometric Properties of the German Version of the Barratt Impulsiveness Scale, Version 11 (Bis-11) for Adolescents. Perceptual and Motor Skills, 112, 353-368. https://doi.org/10.2466/08.09.10.PMS.112.2.353-368

Hu, L. T., \& Bentler, P. M. (1999). Cutoff criteria for fit indexes in covariance structure analysis: Conventional criteria versus new alternatives. Structural Equation Modeling, 6, 1-55. http://dx.doi.org/10.1080/10705519909540118

Hu, L., \& Bentler, P. M. (1995). Evaluating model fit. En Hoyle. R. (Ed.), Structural equation modelling: Concepts, issues and applications (pp.76-99). Thousand Oaks, C.A.: Sage Publications.

Leeman, R. F., Hoff, R. A., Krishnan-Sarin, S., Patock-Peckham, J. A., \& Potenza, M. N. (2014). Impulsivity, sensation-seeking, and part-time job status in relation to substance use and gambling in adolescents. Journal of Adolescent Health, 54, 460-466. https:// doi.org/10.1016/j.jadohealth.2013.09.014

Luengo, M. A., Carrillo de la Peña, M. T. \& Otero, J. M. (1991). The components of impulsiveness: a comparison of the I.7 Impulsiveness Questionnaire and the Barratt impulsiveness Scale. Personality and Individual Differences, 12, 657-667. https://oi.org/10.1016/01918869(91)90220-6

Martínez-Loredo, V., Fernández-Hermida, J. R., Fernández-Artamendi, S., Carballo, J. L., \& García-Rodríguez, O. (2015). Spanish adaptation and validation of the Barratt Impulsiveness Scale for early adolescents (BIS-11-A). International Journal of Clinical and Health Psychology, 15, 274-282. https://doi.org/10.1016/j.ijchp.2015.07.002

Moeller, F. G., Barratt, E. S., Dougherty, D. M., Schmitz, J. M., \& Swann, A. C. (2001). Psychiatric aspects of impulsivity. American Journal of Psychiatry, 158, 1783-1793. https://doi.org/10.1176/appi.ajp.158.11.1783 
Moncada, C., \& Chahín-Pinzón, N. (2019). Factores de riesgo psicosocial y su relación con el funcionamiento y desarrollo neuronal en población en edad escolar. Revista Praxis, 1, 46-49.

Oquendo, M. A., Baca-Garcia, E., Graver, R., Morales, M., Montalvan, V., \& Mann, J. J. (2001). Spanish adaptation of the Barratt impulsiveness scale (BIS-11). European Journal of Psychiatry, 15, 147-55.

Patton, J. H., Stanford, M.S., \& Barratt, E.S. (1995). Factor structure of the Barratt Impulsiveness Scale. Journal of Clinical Psychology, 51, 768-774. https://doi.org/10.1002/1097-4679(199511)51:6<768::AID-JCLP2270510607>3.0.CO;2-1

Pechorro, P., Andershed, H., Ray, J., Maroco, J., \& Gonçalves, R. (2015). Validation of the youth psychopathic traits inventory and youth psychopathic traits inventory: short among incarcerated juvenile delinquents. Journal of Psychopathology and Behavioral Assessment, 37, 576-586. https://doi.org/10.1007/s10862-015-9490-1

Recio, P. (2012). Equivalencia e invarianza de medida entre grupos: análisis factorial confirmatorio vs teoría de respuesta al ítem. (Tesis doctoral). Universidad Complutense de Madrid.

Reise, S. P., Moore, T. M., Sabb, F. W., Brown, A. K., \& London, E. D. (2013). The Barratt Impulsiveness Scale-11: Reassessment of its structure in a community sample. Psychological assessment, 25, 631-642. http://dx.doi.org/10.1037/a0032161

Riaño-Hernández, D., Guillen, A., \& Buela-Casal, G. (2015). Conceptualización y evaluación de la impulsividad en adolescentes: una revisión sistemática. Universitas Psychologica, 14, 1077-1090. http://dx.doi.org/10.11144/Javeriana.upsy14-3.ceia

Saklofske, D. H., \& Eysenck, S. B. G. (1983). Impulsiveness and venturesomeness in Canadian children. Psychological Reports, 52, $147-152$.

Salvo, L., \& Castro, A. (2013). Confiabilidad y validez de la escala de impulsividad de Barratt (BIS-11) en adolescentes. Revista chilena de neuro-psiquiatria, 51, 245-254. http://dx.doi.org/10.4067/S0717-92272013000400003

Secades-Villa, R., Martinez-Loredo, V., Grande-Gosende, A., \& Fernández-Hermida, J.R. (2016). The Relationship between Impulsivity and Problem Gambling in Adolescence. Frontiers in Psychology, 7, 1-7. http://dx.doi.org/ 10.3389/fpsyg.2016.01931

Silva, F., Martorell, M.C., \& Clemente, A. (1987). The I6 questionnaire: Spanish adaptation. Evaluación Psicológica / Psychological Assessment, 3, 55-78.

Stadler, C., \& Janke, W. (2003). Concurrent validity of the German version of SB Eysenck's impulsiveness questionnaire for children. Personality and individual differences, 35, 51-58.

Stahl, C., Voss, A., Schmitz, F., Nuszbaum, M., Tuscher, O., Lieb, K, \& Klauer, K. C. (2013).Behavioral Components of Impulsivity. Journal of Experimental Psychology: General, 143, 850-860. http://dx.doi.org/ 10.1037/a0033981

Stanford, M. S., Mathias, C. W., Dougherty, D. M., Lake, S. L., Anderson, N. E., \& Patton, J. M. (2009). Fifty years of the Barratt Impulsiveness Scale: An update and review. Personality and Individual Differences, 47, 385-395. https://doi.org/10.1016/j.paid.2009.04.008

Vasconcelos, A. G., Malloy-Diniz, L., \& Correa, H. (2012). Sistematic review of psicometric proprierties of Barratt impulseveness scales version 11 (BIS-11). Clinical Neuropsychiatry, 9, 61-74.

Von Diemen, L., Szobot, C. M., Kessler, F., \& Pechansky, F. (2007). Adaptation and construct validation of the Barratt impulsiveness scale (BIS-11) to Brazilian Portuguese for use in adolescents. Revista Brasileira de Psiquiatria, 29, 153-156. http://dx.doi.org/10.1590/ S1516-44462006005000020

Wan, Y., Zhang, M., Jin, F., \& Cheng, Z. (2016). The Chinese Version of the Barratt Impulsiveness Scale-11: Reassessment of Its Psychometric Properties in Three Adolescent Samples. Journal of Psychiatry and Brain Science 1, 1-9. http://dx.doi.org/10.20900/jpbs.20160011

Yao, S., Yang, H., Zhu, X., Auerbach, R. P., Abela, J. R., Pulleyblank, R. W., \& Tong, X. (2007). An examination of the psychometric properties of the Chinese version of the Barratt Impulsiveness Scale, 11th version in a sample of Chinese adolescents. Perceptual and Motor Skills, 104, 1169-82. https://doi.org/10.2466/pms.104.4.1169-1182 